\title{
Is ghrelin a signal for the development of metabolic systems?
}

\author{
Kevin L. Grove and Michael A. Cowley \\ Division of Neuroscience, Oregon National Primate Research Center, Oregon Health \& Science University, Beaverton, Oregon, USA.
}

\begin{abstract}
Ghrelin, produced in the stomach, acts on growth hormone secretagogue receptors (GHSRs) in hypothalamic neurons to potently increase food intake. However, male mice with deletions of ghrelin ( $\mathrm{Gbrl}^{-/-}$mice) or GHSR (Ghsr-/- mice) display normal growth and regulation of food intake. Furthermore, adult $\mathrm{Ghrl}^{-/-}$mice display a normal sensitivity to high-fat diet-induced obesity. These findings from early studies raised the question as to whether the ghrelin system is an essential component for the regulation of food intake and body weight homeostasis. However, recent studies by Wortley et al. and Zigman et al. demonstrate that $\mathrm{Gbrl}^{-/-}$and $\mathrm{Ghsr}^{-/-}$mice are resistant to diet-induced obesity when fed a high-fat diet during the early post-weaning period (see the related articles beginning on pages 3564 and 3573). This commentary highlights 3 key issues raised by these 2 reports: (a) the impact of ghrelin on the development of metabolic systems; (b) the constitutive activity of GHSR; and (c) gender differences in the sensitivity to deletion of the ghrelin signaling system.
\end{abstract}

The growth hormone secretagogue receptor (GHSR) was originally characterized in 1988 by Smith et al. (reviewed in ref. 1) as the receptor for a family of synthetic ligands that stimulated growth hormoneproducing ( $\mathrm{GH}$-producing) pituitary somatotrophs. More than 10 years later, Kojima et al. reported that the endogenous ligand for GHSR was the 28-aa peptide ghrelin, which was produced predominantly in the stomach (2). While ghrelin does indeed stimulate $\mathrm{GH}$ release from the pituitary, this finding was quickly eclipsed by the discovery that this gut peptide also has potent effects on several aspects of energy homeostasis, including the stimulation of food intake, carbohydrate utilization, and decreased lipid metabolism (3). Because chronic ghrelin administration causes weight gain leading to obesity, the ghrelin "pathway" quickly became a potential therapeutic target for the treatment of wasting syndromes. Ghrelin has also been suggested to be the gut signal that initiates a meal, since it is released from the stomach prior to meals, and levels increase greatly in

Nonstandard abbreviations used: AgRP, agouti-related protein; $\mathrm{ARH}$, arcuate nucleus of the hypothalamus; $\mathrm{GH}$, growth hormone; GHSR: growth hormone secretagogue receptor; NPY, neuropeptide Y.

Conflict of interest: The authors have declared that no conflict of interest exists.

Citation for this article: J. Clin. Invest. 115:3393-3397 (2005). doi:10.1172/JCI27211. response to a fast. Ghrelin stimulates food intake through the activation of the orexigenic neuropeptide Y/agouti-related protein (NPY/AgRP) neurons in the arcuate nucleus of the hypothalamus (ARH) $(4,5)$ (Figure 1A). Due to the potential to block this potent orexigenic pathway, antagonists of ghrelin action have been proposed as obesity therapies.

However, several subsequent studies demonstrated that mice in which the genes encoding ghrelin and GSHR had been knocked out did not display the expected lean phenotypes. The animals appeared to have normal growth, regulation of food intake, and energy expenditure (6-8). Furthermore, placing adult ghrelin knockout mice (Ghrl- ${ }^{--}$mice) on a high-fat diet resulted in normal, or near-normal, increases in body weight and adiposity. Thus, deletion of a critical element of this potent feeding system failed to provide the hypothesized protection against diet-induced obesity in adult animals. However, this phenotype might have been predicted, since NPY- and AgRP-knockout and NPY/AgRP doubleknockout mice (9) also display a normal metabolic phenotype under standard laboratory conditions. Was this the end of the ghrelin story? More recent studies have demonstrated that ablation of NPY/ AgRP neurons in the ARH in adult mice, but not infant mice, does cause the predicted hypophagia, indicating that there is developmental compensation in this system $(10,11)$. While this finding reduced the enthusiasm for developing ghrelin antagonists for the treatment of obesity, in this issue of the JCI, 2 separate groups have revisited this question. Wortley et al. (12) and Zigman et al. (13) report, respectively, that deleting ghrelin and GHSR protects against diet-induced obesity in mice started on a high-fat diet in the early postweaning period (4-6 weeks of age). These new studies provide some key insight into the potential role of the ghrelin system in the maintenance and development of metabolic systems and into gender differences in the resistance of $\mathrm{Ghsr}^{-/-}$mice to a highfat diet. The purpose of this commentary is to highlight 3 key concepts raised in these 2 reports: (a) the impact of ghrelin on the development of metabolic systems; (b) the constitutive activity of GHSR; and (c) gender differences in the sensitivity to deletion of the ghrelin signaling system.

Is ghrelin an important signal for the development of metabolic systems? In the studies by Wortley et al. (12) and Zigman et al. (13), male Ghrl-/- and Ghsr ${ }^{-/-}$ mice fed a high-fat diet starting in the early post-weaning period (4-6 weeks of age) displayed an equivalent resistance to dietinduced obesity in comparison to the dietsensitive wild-type animals. Since previous studies had shown that adult $\mathrm{Ghrl}^{-/-}$mice had failed to display a resistance to dietinduced obesity $(6,8)$, Wortley et al. suggest that resistance observed in the young male $\mathrm{Ghrl}^{-/-}$mice is due to early post-weaning exposure to the high-fat diet and is, therefore, a developmental issue. Wortley and colleagues conclude that there might be an interaction of the ghrelin system during the early post-weaning period with the development of neural circuitry in the hypothalamus that controls food intake and that the lack of the ghrelin signal in the developing orexigenic circuits allows the young $\mathrm{Ghrl}^{-/-}$mice to be resistant to the high-fat diet. However, as the animals grow older, the central circuitry may devel- 


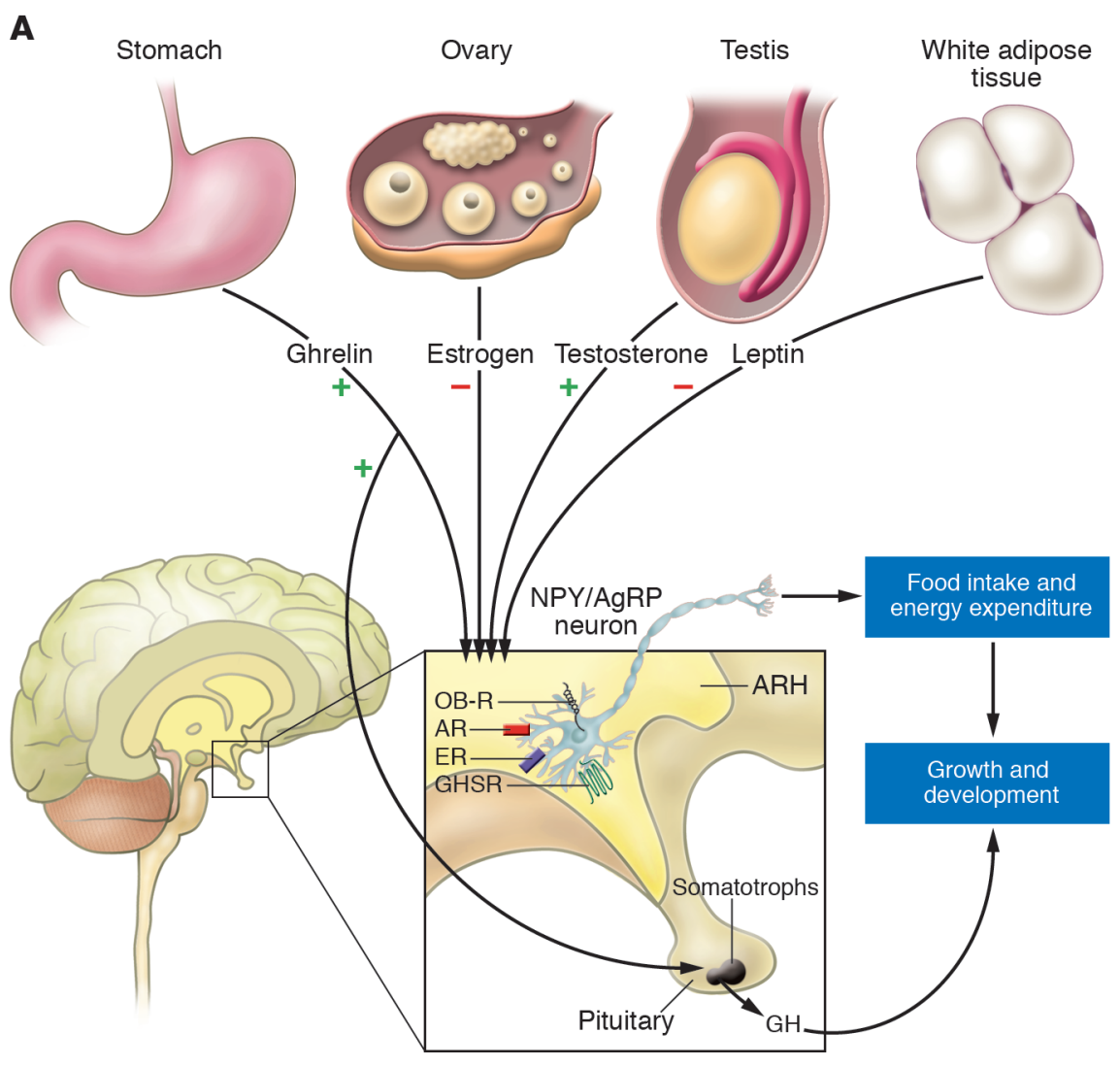

B

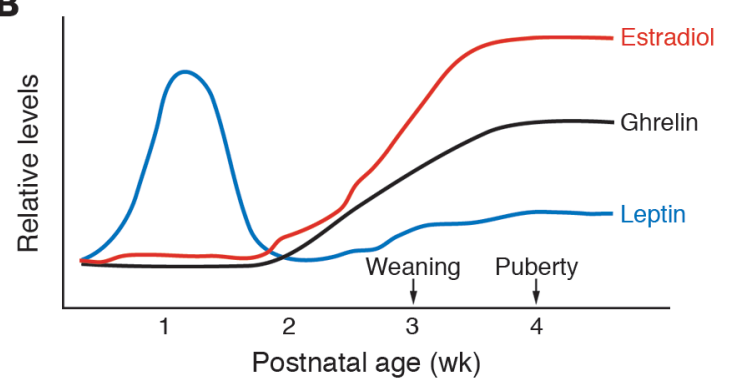

op compensatory pathways to adjust for the loss of the ghrelinergic drive on food intake, thus making the adult $\mathrm{Ghrl}^{-/-}$mice sensitive to diet-induced obesity. Even though the Ghsr ${ }^{-/}$mice displayed a similar resistance to high-fat diet-induced obesity when the studies were initiated at 4 weeks of age, Zigman and colleagues do not raise this possibility of a developmental issue, but rather raise other key possibilities to explain the resistance of these young mice to high-fat diet-induced obesity (as will be discussed below).

What are the potential effects of ghrelin during the critical developmental period in mice? Neurons in the ARH (i.e., NPY/AgRP and $\alpha$-melanocyte-stimulating hormone), which are the key homeostatic feedback regulators of energy balance, start developing axonal projections to efferent target sites near the end of the first postnatal week but don't complete their full projection pattern until the late second to early third postnatal week (14-16). Leptin has been identified as one of the critical signals that initiate the development of these circuits (17), and the natural surge of leptin that occurs during the second postnatal week in the rodent is probably one of the endogenous signals (18) (Figure 1B). Since ghrelin directly excites NPY/AgRP neurons, can it also be a signal for the development of ARH circuits, and do abnormalities in ghrelin signaling during the postnatal period have a long-term impact on homeostatic feedback regulation of energy balance?

\section{Figure 1}

Ghrelin, leptin, and gonadal steroids interact at ARH NPY/AgRP neurons to modulate food intake and energy balance. (A) Ghrelin produced in the stomach directly stimulates ARH NPY/AgRP neurons through the GHSR to increase food intake. Ghrelin can also act directly on pituitary somatotrophs to stimulate the release of $\mathrm{GH}$. Leptin, produced in white adipose tissue, directly inhibits ARH NPY/ AgRP through the leptin receptor (OB-R). Estrogen (released by the ovary) and testosterone (released by the testis) also have direct actions on ARH NPY/AgRP through actions on nuclear estrogen and androgen receptors (ER and AR, respectively). In females, estrogen inhibits food intake and may act together with leptin to counteract the orexigenic effects of ghrelin and promote a lean phenotype. Testosterone stimulates food intake and may act together with ghrelin to promote a more obese phenotype in males. (B) Leptin levels are very low at birth in rodents, show a surge in release toward the end of the second postnatal week, and then slowly increase to reach adult levels between weaning (around 21-23 days of age) and puberty (around 28 days of age). Ghrelin levels are also very low at birth in rodents and show a steady increase leading up to puberty. 
this early age. Surprisingly, in spite of this ability to stimulate $\mathrm{GH}$ release during the postnatal period, chronic daily treatment with ghrelin, from birth to postnatal day 30 , fails to increase growth in both female (19) and male (K.L. Grove et al., unpublished observations) rats.

Although NPY neurons appear responsive to ghrelin in the early postnatal period, it seems unlikely that ghrelin per se is involved in the development of ARH axonal projections. Endogenous ghrelin levels are very low until the third postnatal week (19) (Figure 1B), after the development of ARH circuits; but ghrelin may be involved in development of synaptic inputs into ARH NPY/AgRP neurons. Pinto et al. (20) have demonstrated that ghrelin alters the ratio of excitatory to inhibitory synapses on NPY/AgRP neurons. Alteration of this ratio could have a long-term impact on the excitability of these neurons.

The major question is why adult $\mathrm{Ghrl}^{l^{-/}}$ mice become obese on a high-fat diet, whereas young, post-weaning animals are resistant to the obesity-producing effects of this diet. Clearly, compensatory mechanisms occur in the complete absence of specific orexigenic signals throughout the lifespan of the animals. As mentioned above, NPY- and AgRP-knockout mice, which lack the Npy and Agrp genes throughout their life, or mice that have these neurons ablated soon after birth, develop a nearnormal body weight phenotype $(10,11)$. In addition, like the adult $\mathrm{Ghrl}^{-/-}$mouse, adult $N p y^{-/-}$mice also develop diet-induced obesity that is equivalent to that of wildtype mice (21), suggesting no critical defect in body weight regulation in adults with a deletion of this system. However, mice that have NPY/AgRP neurons ablated as adults become anorexic $(10,11)$, indicating that this orexigenic circuit is critical when the animal goes through normal development with these systems intact. The overall conclusion from all of these studies is that in the absence of specific orexigenic signals, such as NPY, AgRP, or ghrelin, other orexigenic systems are able to compensate to manage normal body weight/energy homeostasis. It should be recognized that in the case of many of the specific gene deletions, such as the Npy, Agrp, or even the $N p y /$ Agrp double knockout, these ARH neurons still exist and release $\gamma$ aminobutyric acid, which itself may be able to maintain the function of these neurons. The strongest evidence of compensatory mechanisms is in the case where ARH NPY/AgRP neu- rons are destroyed at birth but the animals go on to develop normal body weight phenotype $(10,11)$. But it is not known what the compensations in these other orexigenic systems may entail (i.e., increased expression, increased fiber branching, new projections) or when these compensatory mechanisms develop. The data presented by Wortley and colleagues (11), coupled with the findings of previous studies $(6,8)$, may have provided us with critical insight into these questions by demonstrating that early post-weaning $\mathrm{Ghrl}^{-/-}$mice ( 6 weeks of age) are resistant to high-fat diet-induced obesity, but adults (10-20 weeks of age) are sensitive to high-fat diet-induced obesity. The studies by Zigman and colleagues (13) also demonstrate that young $\mathrm{Ghsr}^{-/-}$mice (4 weeks of age) also display resistance to high-fat diet-induced obesity, suggesting that no compensatory mechanisms exist in these animals at this age; however, no studies exist in which these same animals were tested on the high-fat diet as adults. It is worth noting that while there are many examples in which a deletion of a single anorexigenic signal (such as the melanocortin or leptin systems) results in obesity, there are few examples of animals with deletion of a single orexigenic system having a lean phenotype. The most well-known exception to this is melanin-concentrating hormone-null mice, which do display a leaner phenotype as adults (22), indicating that these animals are incapable of developing the compensatory mechanisms.

So what are the mechanisms that allow the young $\mathrm{Ghrl}^{-/-}$mice to resist high-fat diet-induced obesity? In the wild-type animal, the weaning period is characterized by a rise in circulating concentrations of ghrelin and leptin leading up to puberty (around 4 weeks of age). Both leptin and ghrelin can modulate NPY neuronal activity and the synaptic inputs into these neurons in an opposing manner (20). Maybe the critical determinant of neuronal development in this context is the ratio of leptin (inhibitory) to ghrelin (excitatory) signals? Young mice (4-6 weeks of age) lacking ghrelin or GHSR might be exposed to relative hyperleptinemia due to the absence of ghrelin signal, resulting in a chronic unabated inhibitory input into the NPY/AgRP neurons. Thus, a high-fat diet might be better resisted by these mice due to the overall suppression of this orexigenic circuit. Mice that are exposed to high-fat diets later in life might not perceive the same hyperleptinemia because the neural circuits have compensated for the lack of ghrelin signaling to ensure maintenance of body weight. An important next step to interpreting these findings will be to determine whether early exposure to a high-fat diet in ghrelinor GHSR-deficient mice does change the response parameters of these pathways. For this hypothesis to be supported, adult $\mathrm{Ghsr}^{-/-}$mice would need to show normal sensitivity to a high-fat diet and be capable of developing diet-induced obesity. Young $\mathrm{NPy}^{-/-}$or Agrp ${ }^{-/-}$mice should display resistance to weight gain when fed a high-fat diet. It might also be predicted that young $\mathrm{Ghrl}^{-/-}$mice would have different responses to ghrelin, leptin, and peptide YY than adult $\mathrm{Ghrl}^{-/-}$mice. The young $\mathrm{Ghrl}^{-/-}$mice should also display different sensitivity to these inputs when compared with young wild-type mice. More investigation is needed to fully understand the role of ghrelin in the development of metabolic systems, both central and peripheral, as well as to understand the compensatory signals that allow for maintenance of normal body weight and sensitivity to high-fat diets in the absence of potent orexigenic systems.

\section{Does constitutive activity of GHSR contribute to sensitivity to diet-induced obesity?}

Zigman and colleagues (13) suggest an alternative hypothesis to account for the differences between the resistance of their $\mathrm{Gshr}^{-/-}$mice to the high-fat diet and the previously published lack of resistance in $\mathrm{Ghrl}^{+-}$ mice $(6,8)$. They point out that GHSR has constitutive activity (23-26). Thus, some signaling would occur even in the absence of ligand - and hence GHSR-deficient animals would have a complete lack of signaling, while ligand-deficient animals might have residual GHSR signaling. Thus, even in the absence of ghrelin, the basal activity of this receptor could facilitate the development of diet-induced obesity. If the GHSR does exhibit constitutive activity, then in order for a ghrelin antagonist to be effective as an obesity therapy, it may have to also possess inverse agonist properties, to reduce any constitutive activity.

A limitation to the hypothesis that constitutive activity of the GHSR explains the differences in sensitivity to the high-fat diet between $\mathrm{Ghrl}^{-/-}$and $\mathrm{Ghsr}^{-/-}$mice is that the $\mathrm{Ghrl}^{-/-}$mice are resistant to the highfat diet when they are fed the diet during the early post-weaning period (12). If constitutive activity of the GHSR were playing a major role, the $\mathrm{Ghrl}^{-/-}$mice would be 
expected to show at least an intermediate phenotype, i.e., being leaner than wild-type mice but more obese than Ghsr ${ }^{-/-}$mice. However, the male wild-type mice used by Wortley and colleagues displayed the same degree of sensitivity to the high-fat diet as those used by Zigman et al., and the $\mathrm{Ghrl}^{-/-}$ and $G h s r^{-1-}$ mice displayed the same degree of resistance to diet-induced obesity. This is not to suggest that GHSR constitutive activity does not occur, but rather that it does not appear to explain why adult $\mathrm{Ghrl}^{-/-}$ mice are sensitive to the high-fat diet and early post-weaning mice are resistant. To directly compare the effects of deletion of the ligand versus deletion of the receptor, the $\mathrm{Ghsr}^{-/-}$mice need to be exposed to the high-fat diet as adults (10-12 weeks of age). If under these conditions they retain their resistance to high-fat diet-induced obesity, then constitutive activity could be allowing normal development of metabolic systems in the $\mathrm{Ghrl}^{-/-}$mouse, thus allowing it to develop its sensitivity to the high-fat diet as adults.

\section{Gender differences in body weight homeostasis in $\mathrm{Gshr}^{-/-}$mice}

Zigman and colleagues (13) also report some critical gender differences in $\mathrm{Gshr}^{-/-}$ mice, whereby female Gshr-/- mice displayed a leaner phenotype compared with wild-type females when being maintained on either standard or high-fat chow, while male $\mathrm{Gshr}^{-1-}$ mice were indistinguishable from wild-type mice when maintained on a standard chow diet. This gender difference in basal body weight phenotype is consistent with the findings of other investigators who independently generated their own Gshr $r^{-/-}$mice (7). In contrast, Wortley et al. (12) did not find a significant difference between female $\mathrm{Ghrl}^{-/-}$and wildtype mice, but the wild-type female mice from this colony were resistant to high-fat diet-induced obesity, and so direct comparisons are not instructive. However, the preponderance of evidence supports the existence of a gender difference in body weight homeostasis in response to deletion of GHSR. One of the possible sites of interaction between female gonadal steroids (estrogen) and ghrelin is the ARH NPY/AgRP neurons that express both the GHSR and estrogen receptors (27) (Figure 1). Estrogen has similar actions to those of leptin, whereby it decreases food intake and promotes a leaner phenotype (28), with one of the likely actions being inhibition of ARH NPY/AgRP neurons. Thus in the female, leptin and estrogen may act together to promote a leaner phenotype, while ghrelin, which acts on the same ARH NPY/AgRP neurons, may function to counteract the anorexigenic signals to maintain body weight/adiposity. However, in female $\mathrm{Ghsr}^{-/}$mice, which lack the orexigenic ghrelin signaling, the leptin and estrogen actions are unapposed, leading to a leaner phenotype. In contrast, in males, testosterone increases food intake in young animals $(29,30)$ and increases ARH NPY expression (31). Thus testosterone has actions similar to those of ghrelin and may work in conjunction with this gut peptide to promote increased weight gain (Figure 1). Therefore, in male Ghsr ${ }^{-1}$ mice, testosterone is still available to counteract the anorexigenic effects of leptin and also may be able to promote the compensatory mechanisms that develop in adult male Ghsr $r^{-/}$mice to maintain normal body weight and sensitivity to a high-fat diet. These ideas have remained unexplored and need further investigation to truly understand the gender differences in body weight management.

The sexual dimorphism in response to the deletion of the GHSR led Zigman and colleagues (13) to suggest an interesting concept regarding the use of ghrelin antagonists for the treatment of obesity. Female Gshr $r^{-1-}$ mice were leaner when fed either a high-fat or standard chow diet; $\mathrm{Gshr}^{-/-}$males, however, were leaner only when fed a high-fat diet. Thus, it is possible that ghrelin antagonists may be useful in females eating a low-fat or high-fat diet, while they may only help males eating a high-fat diet.

\section{Are ghrelin antagonists good therapeutic targets for the treatment of obesity?}

The studies published in this issue of the JCI $(12,13)$ show that decreased signaling through GHSR and exposure to a high-fat diet in the early post-weaning period can protect against the development of highfat diet-induced obesity. In contrast, previous studies have suggested that there is no protection against diet-induced obesity if the ghrelin-deficient animals are exposed to high-fat food as adults. An important question to be answered is if ghrelin blockade were to be utilized as an antiobesity therapy, to what age does this "early" exposure in a mouse correspond in the timeline of human development? We may assume that a ghrelin antagonist would be of little value if it mimicked what happens when adult GHSR- or ghrelin-deficient mice are fed a high-fat diet. However, there are several reports of ghrelin antagonists having significant effects on energy balance when given to lean and obese adult rats (32-34). Finally, because the GHSR possesses constitutive activity, a ghrelin therapeutic may need to have inverse agonist activity to cause significant changes in energy balance.

The history of antiobesity drug development includes many examples of targets that showed fine promise in rodent studies but failed on the way to the clinic. It is unreasonable to infer from these gene deletion studies that a particular gene product is, or is not, likely to be an effective therapeutic or therapeutic target. Although it may be premature to sell the farm to invest in ghrelin antagonists, these studies provide further hope that ghrelin blockade may have value in the treatment of obesity.

\section{Acknowledgments}

This work was supported by NIH grants DK060685 (to K.L. Grove), DK62202 (to M.A. Cowley), and RR 0163 (to M.A. Cowley and K.L. Grove).

Address correspondence to: Kevin L. Grove, Division of Neuroscience, Oregon National Primate Research Center, Oregon Health \& Science University, 505 NW 185th Avenue, Beaverton, Oregon 97006, USA. Phone: (503) 690-5380 or (503) 690-9503; Fax: (503) 690-5384; E-mail: grovek@ohsu.edu.

1. Smith, R.G., et al. 1997. Peptidomimetic regulation of growth hormone secretion. Endocr. Rev. 18:621-645.

2. Kojima, M., et al. 1999. Ghrelin is a growth-hormone-releasing acylated peptide from stomach. Nature. 402:656-660.

3. Tschop, M., Smiley, D.L., and Heiman, M.L. 2000. Ghrelin induces adiposity in rodents. Nature. 407:908-913.

4. Chen, H.Y., et al. 2004. Orexigenic action of peripheral ghrelin is mediated by neuropeptide Y and agouti-related protein. Endocrinology. 145:2607-2612.

5. Cowley, M.A., and Grove, K.L. 2004. Ghrelin - satisfying a hunger for the mechanism. Endocrinology. 145:2604-2606.

6. Sun, Y., Ahmed, S., and Smith, R.G. 2003. Deletion of ghrelin impairs neither growth nor appetite. Mol. Cell. Biol. 23:7973-7981.

7. Sun, Y., Wang, P., Zheng, H., and Smith, R.G. 2004. Ghrelin stimulation of growth hormone release and appetite is mediated through the growth hormone secretagogue receptor. Proc. Natl. Acad. Sci. U. S. A. 101:4679-4684.

8. Wortley, K.E., et al. 2004. Genetic deletion of ghrelin does not decrease food intake but influences metabolic fuel preference. Proc. Natl. Acad. Sci. U. S. A. 101:8227-8232. 
9. Qian, S., et al. 2002. Neither agouti-related protein nor neuropeptide $\mathrm{Y}$ is critically required for the regulation of energy homeostasis in mice. Mol. Cell. Biol. 22:5027-5035.

10. Gropp, E., et al. 2005. Agouti-related peptideexpressing neurons are mandatory for feeding. Nat. Neurosci. 8:1289-1291.

11. Luquet, S., Perez, F.A., Hnasko, T.S., and Palmiter, R.D. 2005. NPY/AgRP neurons are essential for feeding in adult mice but can be ablated in neonates. Science. 310:683-685.

12. Wortley, K.E., et al. 2005. Absence of ghrelin protects against early-onset obesity. J. Clin. Invest 115:3573-3578. doi:10.1172/JCI26003.

13. Zigman, J.M., et al. 2005. Mice lacking ghrelin receptors resist the development of diet-induced obesity. J. Clin. Invest. 115:3564-3572. doi:10.1172/ JCI26002.

14. Grove, K.L., Allen, S., Grayson, B.E., and Smith, M.S. 2003. Postnatal development of the hypothalamic neuropeptide Y system. Neuroscience. 116:393-406.

15. Grove, K.L., and Smith, M.S. 2003. Ontogeny of the hypothalamic neuropeptide Y system. Physiol. Behav. 79:47-63.

16. Bouret, S.G., Draper, S.J., and Simerly, R.B. 2004. Formation of projection pathways from the arcuate nucleus of the hypothalamus to hypothalamic regions implicated in the neural control of feeding behavior in mice. J. Neurosci. 24:2797-2805.

17. Bouret, S.G., Draper, S.J., and Simerly, R.B. 2004. Trophic action of leptin on hypothalamic neurons that regulate feeding. Science. 304:108-110.

18. Ahima, R.S., and Hileman, S.M. 2000. Postnatal regulation of hypothalamic neuropeptide expression by leptin: implications for energy balance and body weight regulation. Regul. Pept. 92:1-7.

19. Hayashida, T., et al. 2002. Ghrelin in neonatal rats: distribution in stomach and its possible role. J. Endocrinol. 173:239-245.

20. Pinto, S., et al. 2003. Rapid re-wiring of arcuate nucleus feeding circuits by leptin. Science. 304:110-115.

21. Hollopeter, G., Erickson, J.C., and Palmiter, R.D. 1998. Role of neuropeptide Y in diet-, chemicaland genetic-induced obesity of mice. Int. J. Obesity. 22:506-512.

22. Shimada, M., Tritos, N.A., Lowell, B.B., Flier, J.S., and Maratos-Flier, E. 1998. Mice lacking melaninconcentrating hormone are hypophagic and lean. Nature. 396:670-674

23. Holst, B., Brandt, E., Bach, A., Heding, A., and Schwartz, T.W. 2005. Nonpeptide and peptide growth hormone secretagogues act both as ghrelin receptor agonist and as positive or negative allosteric modulators of ghrelin signaling. Mol. Endocrinol. 19:2400-2411.

24. Holst, B., et al. 2004. Common structural basis for constitutive activity of the ghrelin receptor family. J. Biol. Chem. 279:53806-53817.

25. Holst, B., Cygankiewicz, A., Jensen, T.H., Ankersen, M., and Schwartz, T.W. 2003. High constitutive signaling of the ghrelin receptor - identification of a potent inverse agonist. Mol. Endocrinol. 17:2201-2210.

26. Lall, S., et al. 2004. Physiological studies of transgenic mice overexpressing growth hormone $(\mathrm{GH})$ secretagogue receptor $1 \mathrm{~A}$ in $\mathrm{GH}$-releasing hormone neurons. Endocrinology. 145:1602-1611.

27. Sar, M., Sahu, A., Crowley, W.R., and Kalra, S.P. 1990. Localization of neuropeptide-Y immunoreactivity in estradiol-concentrating cells in the hypothalamus. Endocrinology. 127:2752-2756.

28. Eckel, L.A. 2004. Estradiol: a rhythmic, inhibitory, indirect control of meal size. Physiol. Behav. 82:35-41

29. Nunez, A.A., and Grundman, M. 1982. Testosterone affects food intake and body weight of weanling male rats. Pharmacol. Biochem. Behav. 16:933-936.

30. Nunez, A.A. 1982. Dose-dependent effects of testosterone on feeding and body weight in male rats. Behav. Neural. Biol. 34:445-449.

31. Sohn, E.H., Wolden-Hanson, T., and Matsumoto, A.M. 2002. Testosterone (T)-induced changes in arcuate nucleus cocaine-amphetamine-regulated transcript and NPY mRNA are attenuated in old compared to young male brown Norway rats: contribution of $\mathrm{T}$ to age-related changes in cocaineamphetamine-regulated transcript and NPY gene expression. Endocrinology. 143:954-963.

32. Kobelt, P., et al. 2005. Anti-ghrelin SPIEGELMER NOX-B11 inhibits neurostimulatory and orexigenic effects of peripheral ghrelin in rats. Gut. doi:10.1136/gut.2004.061010.

33. Helmling, S., et al. 2004. Inhibition of ghrelin action in vitro and in vivo by an RNA-Spiegelmer. Proc. Natl. Acad. Sci. U. S. A. 101:13174-13179.

34. Beck, B., Richy, S., and Stricker-Krongrad, A. 2004. Feeding response to ghrelin agonist and antagonist in lean and obese Zucker rats. Life Sci. 76:473-478.

\title{
Expanding the immunotherapeutic potential of minor histocompatibility antigens
}

\author{
Eric Spierings and Els Goulmy
}

Department of Immunohematology and Blood Transfusion, Leiden University Medical Center, Leiden, The Netherlands.

\begin{abstract}
Minor histocompatibility antigens (mHAgs) selectively expressed by cells or cell subsets of the hematopoietic system are targets of the $T$ cell-mediated graft-versus-leukemia response that develops following allogeneic hematopoietic stem cell transplantation (HSCT) for the treatment of hematological malignancies. This observation has served as the rationale for utilizing mHAg-specific immunotherapy for the treatment of particular patients. However, at present, only a select and small number of patients could potentially benefit from mHAg-based immunotherapy. A report from de Rijke et al. in this issue of the JCI describes a new hematopoietic lineage-specific HLA-B7-restricted mHAg associated with remission of chronic myeloid leukemia (see the related article beginning on page 3506). This result represents another example of an mHAg-mediated graft-versus-leukemia response, thereby expanding the number of patients eligible for $\mathrm{mHAg}$-based immunotherapy in the setting of HSCT.
\end{abstract}

Nonstandard abbreviations used: GVHD, graft-versus-host disease; GVL, graft-versus-leukemia; HSCT, hematopoietic stem cell transplantation; LRH-1, lymphoid-restricted histocompatibility antigen-1; mHAg, minor histocompatibility antigen.

Conflict of interest: The authors have declared that no conflict of interest exists.

Citation for this article: J. Clin. Invest. 115:3397-3400 (2005). doi:10.1172/JCI27094.

\section{Characteristics of minor histocompatibility antigens applicable for immunotherapy}

Minor histocompatibility antigens (mHAgs) were originally defined in mice by characterization of in vivo rejection responses to skin grafts and tumors exchanged between mice of different inbred strains $(1,2)$. Simultane- ous with the discovery that matching HLA antigens are necessary for optimal success of allogeneic BM transplantation (3), clinical results demonstrated the powerful alloimmune reactions against mHAgs. In an HLA-matched hematopoietic stem cell transplantation (HSCT) setting, mHAg disparities between recipient and donor can lead to graft-versus-host disease (GVHD) (4) or graft rejection (5). Aside from these detrimental effects, the mHAg-induced alloimmune response also causes the curative graft-versus-leukemia (GVL) effect. Since mHAg-specific $T$ cells are involved in both GVHD and GVL, dissecting the role of these cells in the immunobiology of GVHD and GVL has proven challenging. The first indication that led us to propose the use of mHAgs as immunotherapeutic tools in HSCT (6) was provided by the results of in vitro studies showing differential modes of recognition of various cell types by mHAgspecific CTLs, i.e., ubiquitous or hemato- 